\title{
Lipid Profile and Differential Lipids in Serum Predict Severity of Community-Acquired Pneumonia: A Prospective, Multi-Centre Study
}

\section{Li Chen}

Peking University People's Hospital

\section{Yali Zheng}

Xiang'an Hospital of Xiamen University

\section{Lili Zhao}

Peking University People's Hospital

\section{Ying Zhang}

Peking University People's Hospital

\section{Lu Yin}

Peking University People's Hospital

\section{Yukun He}

Peking University People's Hospital

\section{Xinqian Ma}

Peking University People's Hospital

YuXu ( $\boldsymbol{\nabla}$ pkuph_xuyu@163.com)

Peking University People's Hospital

Zhancheng Gao ( $\boldsymbol{\sigma}$ zcgao@bjmu.edu.cn )

Peking University People's Hospital https://orcid.org/0000-0002-8495-9221

\section{Research}

Keywords: Community acquired pneumonia, Lipidomic profile, Severity, Mortality

Posted Date: March 6th, 2020

DOI: https://doi.org/10.21203/rs.3.rs-16271/v1

License: (c) (1) This work is licensed under a Creative Commons Attribution 4.0 International License.

Read Full License 


\section{Abstract}

Background: This study aimed to characterize the lipidomic responses to community-acquired pneumonia (CAP), and validate the effectiveness of these lipids as indicators of the severity of the CAP as well as of the risk of death in severe CAP.

Methods: Lipidomic profiles of serum were generated using ultra high-performance liquid chromatography with tandem mass spectrometry (UHPLC-MS/MS). Principal component analysis (PCA) and supervised orthogonal partial least squares discriminant analysis (OPLS-DA) was implemented to distinguish between the classes of samples and to identify the differentially expressed variables. The area under the receiver operating characteristic curve of the significant differential lipids was compared. Spearman's rank correlation test and multiple linear regression (MLR) analysis were applied to further explore the putative differentially expressed lipids correlation with clinical parameters. Kaplan-Meier methods were used to build 30-day survival curves, and survival rates were compared using the log-rank test.

Results: A total of 6 categories and 509 lipid species were detected in the positive ion mode using UPLCMS/MS. In the negative ion mode, 3 categories and 195 lipid species were detected. According to the screening criteria, 5 lipids in total were selected as target lipids, which are PC (16:0_18:1), PC (18:2_20:4), PC (20:5_18:2), PC (36:4) and PC (38:6). Area under curves (AUC) for all five li $p$ ids were superior to PSI (0.749, 0.550-0.892) and CURB-65 (0.772, 0.575-0.908). PC (18: 2_20: 4), PC (38: 6) and PC (36: 4) was negatively related to FiO 2 and PC (18:2_20: 4) was inversely correlated with the PCT. As the relative abundance of PC (16:0_18:1), PC (36: 4), PC (20: 5_18: 2) and PC (38: 6) decreased, the length of hospital stay significantly extended. There was a statistically significant difference in the probability of 30-day mortality between the high-abundance and low-abundance groups of PC (16: 0_18: 1), PC (36: 4) and PC (20: 5_18: 2) (og-rank $p<0.05)$.

Conclusion: Our research suggests that the serum lipidomics approach of the UHPLC-MS/MS platform can be used to reveal lipid changes during CAP and establish lipid profiles related to disease severity.

Trial registration: ClinicalTrials.gov, NCT03093220. Registered on 28 March 2017 (retrospectively registered).

\section{Introduction}

Community acquired pneumonia (CAP) remains a major health concern worldwide with substantial morbidity and mortality, especially among geriatric populations [1]. Moreover, the severity of clinical manifestations of CAP significantly varies [2-3]. Because of the diversity in clinical conditions and the lag in a clear definition of the causative pathogen, timely identification and diagnosis, accurate assessment, and prediction of disease severity are critical for effectively managing individuals with CAP [4]. 
Lipids are the major components of alveolar surfactant [5]. In recent years, the rapid development of lipidomics has enabled us to gain new insights into the mechanism of disease development [6-7]. Lipids play a critical role in cellular energy storage, structure, and signalling [8]. They are not only considered as the components of membranes and source, but also act as an indispensable factor in the immune response by organizing signalling complexes in cellular membrane [9]. Studies have confirmed that lipids act as important inflammatory mediators during infection process [7, 9-10]. Changes in lipid components in serum or plasma can occur during acute lung injury, sepsis, bacteraemia, and viral infections [11-14]. Biomarkers play an important role in promoting early severity assessment of the disease and predicting treatment response [15]. In recent years, the focus of the discovery of CAP biomarkers has increasingly turned to molecular expression profiling, which is employed in the initial diagnosis and clinical treatment of pneumonia [16-17]. Bioactive lipids located further downstream in the biological system can better reflect the body's redox balance, oxidative stress, signalling, apoptosis, and inflammation than biological and gene expression biomarkers in body fluids; therefore, it provides more relevant and richer features in CAP [17]. Considering these intimate connections between lipids and processes relating to the pathogenesis of pneumonia, it is conceivable that changes in plasma lipid populations can be mined for biomarker discovery. However, none of the studies so far have focused on lipidomics changes in the serum of patients with different degrees of CAP. Moreover, no biologically active lipid has been found to play a monitoring role in the process of CAP exacerbation, and assisting in the diagnosis and prognosis of disease at the early stage.

In the current study, untargeted lipidomics research using high-performance liquid chromatography-mass spectrometry (HPLC-MS) was performed to identify CAP-related lipidomic signatures. The relationship between the identified lipids and the severity of CAP was then investigated. The characterization of the lipidomics response to CAP patients can provide new perspectives on the underlying mechanisms of pathogenesis and potential avenues for diagnostic and therapeutic treatments.

\section{Materials And Methods}

\section{Study population}

This prospective, multi-centre, and observational study was performed from January 2017 to October 2018 among patients hospitalized at the Peking University People's Hospital (PKUPH), Fujian Provincial Hospital, Sichuan University West China Hospital, Shanghai Pulmonary Hospital, The Second Hospital of Jilin University, and Tibet Autonomous Region People's Hospital. This study was registered at ClinicalTrials.gov (NCT03093220) and was endorsed by the Institutional Review Board of the PKUPH. All CAP patients were recruited from the respiratory medicine department or intensive care unit (ICU). All participants provided written informed consent prior to the collection of any data.

Inclusion criteria for CAP were [18] evidence of pulmonary infiltrate on chest, a chest radiograph showing either a new patchy infiltrate, leaf or segment consolidation, ground glass opacity, or interstitial change. Additionally, the inclusion criteria includes at least one of the following signs: (a) the presence of cough, 
purulent sputum production, and dyspnoea; (b) fever (core body temperature $>38.0^{\circ} \mathrm{C}$ ); (c) auscultatory findings of abnormal breath sounds and rales; or (d) leucocytosis or leukopenia (peripheral white blood cell counts $>10 \times 10^{9} / \mathrm{L}$ or $<4 \times 10^{9} / \mathrm{L}$ ), and if symptom onset began in communities. Severe CAP (SCAP) was defined according to the presence of at least one major criterion or at least three minor criteria published by the American Thoracic Society in 2007 [2]. Minor criteria include respiratory rate $\geq 30$ breaths/minute, $\mathrm{PaO}_{2} / \mathrm{FiO}_{2}$ ratio $\leq 250$, multipolar infiltrates, confusion or disorientation, uraemia (BUN level $\geq 20 \mathrm{mg} / \mathrm{dL}$ ), leukopenia (WBC count $<4000$ cells $/ \mathrm{mm}^{3}$ ), thrombocytopenia (platelet count $<100,000$ cells $/ \mathrm{mm}^{3}$ ), Hypothermia (core temperature $<36^{\circ} \mathrm{C}$ ), and hypotension requiring aggressive fluid resuscitation. Major criteria are as follows: invasive mechanical ventilation and septic shock with the need for vasopressors.

The exclusion criteria were age $<18$ years, or the presence of any of the following: women who were pregnant or lactating, patients who had surgery within 3 months before onset, patients with evidence of nosocomial infection, and immunosuppressive condition. Furthermore, patients with malignant tumour, chronic neurological diseases (e.g. Parkinson's disease, multiple system atrophy), end-stage renal or liver disease, and active tuberculosis, or pulmonary cystic fibrosis were excluded as well.

Demographic information was obtained using standard forms (including age, gender, smoking history, underlying diseases, complications, symptoms, signs, laboratory test results, and clinical treatment methods) for general participants. Patient outcomes were evaluated at discharge and assessed through structured telephone interviews 30 days after enrolment. The outpatient department of PKUPH conducted routine health checks on healthy volunteers and collected basic information.

\section{Blood sample collection and preparation}

A total of $5 \mathrm{~mL}$ of fasting peripheral venous blood was drawn from adult CAP patients during the first 72 hours of hospital admission, injected into the sterile pro-coagulation tubes, and allowed to stand at room temperature for 30 minutes, followed by centrifugation and was aliquoted into 2-3 tubes. The resulting serum samples were stored at $-80^{\circ} \mathrm{C}$ for processing.

Serum samples were thawed on ice at the time of assay, $100 \mu \mathrm{L}$ fluid was taken and centrifuged at $14,000 \mathrm{~g}$ at $4{ }^{\circ} \mathrm{C}$ for 20 minutes, and then the supernatant was transferred to a new Eppendorf tube, and $400 \mu \mathrm{L}$ methanol was added to the supernatant. This was vortexed repeatedly and allowed to stand for three times, followed by centrifugation after full extraction. At this time, the solution was separated into two layers, and the lower organic phase was pipetted into a new $1.5 \mathrm{ml}$ Eppendorf tube and dried before storage in the $-80^{\circ} \mathrm{C}$ freezer until untargeted LC-MS analysis. To monitor the repeatability and stability of the analysis system, a pooled quality control (QC) sample solution was prepared by combining $10 \mu \mathrm{L}$ aliquots from each sample and extracted as above.

\section{Untargeted HPLC-MS analysis and lipids identification}


The Ultimate 3000 UHPLC (Dionex) system coupled with Q Exactive (Orbitrap) mass spectrometer (Thermo Fisher, CA) was used to detect the sample to be tested, and detection was performed in the positive and negative ion scanning modes, respectively. Before analysing the experimental samples, six QCs need to be injected to achieve stability for the instrument. The analysis sequence between QC samples and test samples was randomized between every 10 experimental samples.

TraceFinder 3.2 software (Thermo Fisher Scientific, USA) was used to collect raw data, and perform denoising, baseline correction, peak alignment, peak signal detection, and segment integration processing on the mass spectrum. Then, according to its precursor and characteristic mass, the data was compared to the local self-built database in "screening" mode and quantified in "Quan" mode. Finally, mass spectrum data matrix composed of mass to charge ratio $(\mathrm{m} / \mathrm{z})$, retention time (RT), and peak abundance was obtained.

\section{Analysis and processing of untargeted UHPLC-MS/MS lipidomics data}

It was necessary to pre-process the data to reduce the interference information and facilitate the mining of more meaningful biological information. To remove noise and improve data quality a lipid was included in the next analysis if it had a non-zero value for at least $80 \%$ in the samples of any group. The missing values in the data matrix needed to be filled. Considering that most of the missing values were caused by low-abundance compounds (that is, below the detection threshold), we replaced the missing values with half the minimum value of the detected value of the compound in the original data. We standardized the data to correct for the effects of factors such as individual differences or differences in sample collection or processing on absolute compound concentrations. Normalization is then performed to eliminate inconsistencies in concentration between different compounds. The obtained normalized data were further analysed. The above data pre-processing was performed using MetaboAnalyst 4.0 [19].

\section{Multidimensional statistical analysis}

The resulting normalized data were imported into SIMCA-P 14.0 (Umetrics, Sweden) for multivariate variable analysis (MVA). First, a principal component analysis (PCA) model was established to observe the overall distribution of samples in each group, explore possible factors affecting sample aggregation, and judge outliers. Second, according to the clinical grouping, an orthogonal partial least squares discriminant analysis (OPLS-DA) model was established for the samples to extract the different information between the groups, and the variable importance on projection (VIP) value was obtained. The VIP value greater than 1 indicated that the between-group differences were greater than the within-group differences. We used the R2X, R2Y, and Q2 parameter values to determine the quality of the OPLS-DA model. The quality of the MVA models was evaluated by cross-validation analysis of variance (CVANOVA) and permutation test (500 iterations) [20].

\section{Statistical analysis}


Categorical variables are expressed as numbers (percentages) and analysed using a chi-square test or Fisher's exact test. The Kolmogorov-Smirnov test was used to evaluate the distribution of continuous variable data. Continuous variables are expressed as mean \pm standard deviation (mean \pm SD) when they met the normal distribution, were analysed using Student's t-test or analysis of variance (ANOVA) with post hoc Tukey HSD test. Continuous nonparametric data are presented as median and interquartile ranges (IQR, 25th and 75th percentiles) and was analysed using Mann-Whitney $\mathrm{U}$ or Kruskal-Wallis $\mathrm{H}$ test, as appropriate. The heat map with a Euclidean distance measure of relative intensity of metabolites (logarithmic scale) and a Pearson's correlation heat map was generated using MetaboAnalyst 4.0 (http://www.metaboanalyst.ca/; Wishart Research Group, University of Alberta, USA) [19]. Potential small molecule biomarkers screened by lipidomics were analysed by receiver operating characteristic curve (ROC), and the area under the curve (AUC) and 95\% confidence interval (95\% Cl) was calculated. The twotailed Spearman's rho test correlation coefficient $(r)$ was calculated to evaluate the strength and direction of the linear relationship between the putative lipid biomarker and clinical indicators. Furthermore, to adjust the multicollinearity inherent in lipidomics data, a stepwise approach was used to perform multiple linear regression (MLR) analysis. The Kaplan-Meier method was applied to establish a 30-day survival curve, and logarithmic rank tests were utilized to compare survival rates. Cox proportional hazards regression analysis was exploited to analyse the effect of lipid biomarkers on 30-day survival.

Statistical tests were performed using the SPSS statistics version 19.0 (IBM, NY, USA) and MedCalc Software version 15.8 (MedCalc Software, Ostend, Belgium).

\section{Results}

\section{Demographic and clinical characteristics of participants}

The final study population consisted of 28 patients with CAP (15 NSCAP and 13 SCAP) and 20 age, sexmatched healthy controls $(\mathrm{HC})$ at multiple centers including 6 hospitals in China. As indicated in Table 1, there was a range of aetiologies of CAP including bacteria, virus, and fungus, which was typical for a heterogeneous CAP patient population. Particularly, there were no significant differences in age, sex, basal metabolic rate, smoking history, underlying disease among the three groups $(p>0.05)$. However, in laboratory test, the inflammatory response related indicators such as the percentages of neutrophil (NE\%), lymphocyte (LY\%), and monocyte (MO\%), also, the levels of white blood cell (WBC), neutrophil (NE) and lymphocyte(LY) were all significantly different in the SCAP compared with that in the NSCAP (all $p<$ 0.05). The levels of serum C-reactive protein (CRP) and procalcitonin (PCT) were both greater in the SCAP group $(p<0.05)$. In terms of physical examination, respiratory frequency of SCAP patients dramatically increased $(p<0.05)$. CURB-65, PSI was all prominently higher in the severe CAP group than in the NSCAP group $(p<0.05)$. Comparing the detection of pathogens between the two groups, we found that the detection rate of bacteria in patients with SCAP was higher. In addition, hospitalization days and 30-day mortality were both substantially higher in patients with SCAP than in those with NSCAP $(p<0.05)$ (Table 1). 


\section{Global lipidomic profiles of human serum}

Serum lipidomic profiles of 48 subjects were generated through untargeted lipidomic profiling analysis using HPLC-MS/MS. Overall, a total of 6 categories (509 species) lipid were detected in the election spray ionization positive (ESI+) mode. Top 3 dominant categories comprised over $99 \%$ of the total lipid signal, including glycerol-phospholipids (GP, 44.78\%), glycerolipids (GL, 38.19\%), and sphingolipids (SP, 16.06\%). The lipid profile of each of the three groups was different. The relative abundance of GP, GL, and SP in the CAP group (NSCAP and SCAP) were decreased compared with that in HC. In addition, as the severity of the CAP increases, GL continued to decrease, while the relative abundance of GP and SP increased slightly. The relative abundances of sterol lipids (ST), prenol lipids (PR), and fatty acyls (FA) were also higher in the CAP group than $\mathrm{HC}$.

In the election spray ionization negative (ESI-) mode, 195 lipid species were detected, and FA was more effectively recognized. Only three types of compounds were detected in the negative ion mode, namely GP, SP, and FA. They accounted for $57.14 \%, 25.83 \%$, and $17.03 \%$, respectively. Where subclasses such as phosphatidylcholine (PC, 47.14\%), sphingomyelin (SM, 22.35\%) and free fatty acid (FFA, 17.03\%) contributed $74.05 \%$ to the total lipid signal. Similarly, there are numerous differences in serum lipid profiles between NSCAP, SCAP and HC. Compared with HC, the relative abundance of NSCAP and SCAP group was decreased, and as the condition worsened, it continued to decline.

\section{Multivariate models established by untargeted lipidomics analysis.}

PCA is an effective mean for classifying data, detecting outliers, and validating the stability and reproducibility of an analytical method. All identified lipids were subjected to PCA using MetaboAnalyst 4.0 to explore the major effects that potentially drive the differences in lipid profiles in CAP patients (NSCAP and SCAP) and HC (Fig. 1a). The optimal PCA model contains eight components. First and second components explain $24.6 \%$ and $12.2 \%$ of the variability between samples, respectively. The value of R2 (cum) and Q2 (cum) (0.674 and 0.449), respectively, represented the fit and predicted power of the model. All QC samples were tightly clustered indicating that this instrument has good repeatability and stability. As shown in Fig. 1a, no obvious abnormal points were found in all serum samples. Importantly, the obvious intra-group clustering and inter-group separation between three groups suggested that whether CAP or SCAP, the patient's serum lipid profile has changed significantly.

OPLS-DA analysis identified the biggest variation in lipid profiling using a few orthogonal latent variables. In order to further eliminate the interference factors of the disease and maximize the extraction of the information on the differences in lipid mass spectra between different groups, we used a supervised clustering method to verify the OPLS-DA model of the serum samples of the subjects. To prevent over fitting, we performed a permutation test (500 iterations) on those models. The OPLS-DA score plot showed obvious discriminatory trend between both CAP group versus HC, NSCAP versus HC, SCAP versus $\mathrm{HC}$, and SCAP versus SCAP (Fig. 1: b-e). The CV-ANOVA $p$-values for all models were less than 0.0001 , indicating that all the differences between the groups were significant. The Q2Y of all models was higher than 0.9 , which revealed the model has excellent interpretation ability and superior predictive 
power (Q2>0.5) (Table 2). After the 500-it iteration of the permutation test, the R2 and Q2 values were smaller than the original model (Table 2), and the Q2 regression line was less than 0 in the $\mathrm{Y}$-axis intercept (Additional file 1: Fig. S1), which proved that the model was robust and there was no over-fitting. Notably, in the OPLS-DA model, the predictability of separating SCAP from the HC (Q2 $=0.848$ ) was better compared with separating NSCAP from the HC $(\mathrm{Q} 2=0.703)$. That is, compared with NSCAP, more changes were observed in SCAP and better isolation from the HC.

\section{Differential lipids associated with CAP disease and acute exacerbations}

In order identify lipids with significant differences between CAP versus HC and NSCAP versus SCAP, we normalized the relative peak intensity data of all lipids detected and performed univariate analysis. A total of 295 lipids were statistically different (FDR adjusted $p$-value $<0.05$ ) between the CAP and HC. The VIP score was used to quantify the contribution of each lipid to the overall separation between two groups in the OPLS-DA model. There were 226 lipids with VIP values greater than 1 between the CAP and HC. Compared with NSCAP and SCAP at the same time, 297 lipids had statistical differences, and VIP of 246 lipids exceeded 1.

Interestingly, we found that not only did many lipids differ significantly in the CAP group, but the relative abundance also changed significantly, as the disease worsened. In order to screen out the highest potential lipid that could distinguish CAP from $\mathrm{HC}$ and assess the severity at the early stage. VIP> 1 and FDR $<0.05$ were set as the selection criteria. A total of 85 lipids met the screening criteria both in the comparison of CAP versus $\mathrm{HC}$ and NSCAP versus SCAP. It is a remarkable fact that most of the differential lipids were continuously up or down regulated in the CAP and SCAP group (Fig. 2a).

\section{Receiver operating characteristic (ROC) curve analysis}

The ROC was applied according to above results for the area under the curve (AUC) and sensitivity/specificity at the best cut-off points. We further selected lipids with AUC greater than 0.85 in the two comparisons as target lipids. There are 5 lipids in total, which are PC (16:0_18:1), PC (18:2_20:4), PC (20:5_18:2), PC (36:4), and PC (38:6). AUC (95\% Cl) for all five lipids above were superior to PSI (0.749, 0.550-0.892) and CURB-65 (0.772, 0.575-0.908), so they might be considered as a potential panel of lipid biomarkers for assessing the severity of CAP (Additional file 1: Fig. S2). Simultaneously, the relative abundance of PC (18: 2_20: 4), PC (20: 5_18: 2), PC (36: 4), and PC (38: 6) was significantly lower in the CAP group than in the $\mathrm{HC}$ group, and their abundance continued to decrease as the disease worsened. However, the relative abundance of PC (16: 0_18: 1) showed the opposite trend (Fig. 2b-f).

\section{Correlation between putative biomarkers and clinical indicators}

Since these clinical indicators showed abnormal distribution, Spearman's rank correlation test was applied to further explore if the target lipids correlated with the clinical parameters: WBC, NE, LY, LY (\%), $\mathrm{MO}(\%)$ and $\mathrm{NE}(\%)$ in serum, CRP, PCT, $\mathrm{FiO}_{2}, \mathrm{PaO}_{2} / \mathrm{FiO}_{2}, \mathrm{CURB}-65$, and PSI. Owing to the lack of 
laboratory test data in the $\mathrm{HC}$, we calculated the correlation between clinical indicators and differential lipids in the NSCAP and SCAP (Additional file 1: Fig. S3; Additional file 2: Table S1-S2).

According to the results of correlation analysis, target lipids mentioned above were related to indices of infection. Considering the strong co-linearity between those lipids, MLR analysis was conducted to evaluate the biochemical indices, which were independently correlated to lipids. Finally we identified that PC (18: 2_20: 4), PC (38: 6) and PC (36: 4) were negatively related to $\mathrm{FiO}_{2}$ after $p$ value adjustment. In addition, PC (18: 2_20: 4) was inversely correlated with PCT (Fig. 3).

\section{Putative lipids monitor disease progression and assess prognosis}

Further, to investigate whether the putative biomarker can predict the prognosis of patients, we utilized the SCAP diagnostic threshold, which was the cut-off value, of the grouping criterion according to the ROC calculation results. Depending on the relative abundance of those lipids in plasma, patients were redivided into low-abundance group and high-abundance group. Immediately afterwards, we calculated whether there were statistical differences in hospitalization days between the two groups. The results demonstrate that the hospitalization days in the two groups of PC (16:0_18:1), PC (36:4), PC (20:5_18:2), and PC (38:6) were statistically significant ( $p<0.05$, Fig. 4).

Kaplan-Meier curves were used to determine if there were statistically significant differences in mortality between the high-abundance group and low-abundance group of PC (16: 0_18: 1), PC (36: 4) and PC (20: 5_18: 2) ( $p=0.0072,0.0219$, and 0.0491 respectively) (Fig. 5). Obviously, by monitoring the fluctuations of the above lipids, it is possible to effectively monitor the evolution of the disease and systematically assess the patient's prognosis early.

\section{Discussion}

In this prospective, multi-centre study, we used UHPLC-MS / MS technology to conduct exploratory studies and describe lipid mass spectrometry characteristics in 48 human serum samples, including 28 CAP and $20 \mathrm{HC}$. We found that the lipid profiles of the HC, NSCAP, and SCAP were significantly different in both positive and negative ion mode. The relative abundance of GP, GL, and SP in the CAP group was decreased. As the severity of the CAP increases, GL continued to decline, while the relative abundance of GP and SP increased slightly in the positive ion mode. While in ESI- mode, the levels of GP, SP and FA was decreased compared to those in controls, and as the condition worsened, they continued to decline. Untargeted lipidomic analysis could clearly discriminate CAP patients from HC, as well as the NSCAP group from the SCAP, which further suggested that CAP and disease exacerbation caused significant fluctuations in lipidomic biochemical homeostasis. We further selected 5 lipids with AUC greater than 0.85 in the two comparisons of CAP versus $\mathrm{HC}$ and NSCAP versus SCAP as target lipids. They were considered putative biomarkers for early recognition of CAP, closely related to the severity of CAP, and might represent a panel of potential small molecule lipids for assessing CAP severity. We also identified individual lipids at the molecular level PC (18:2_20:4), PC (36:4) and PC (38:6) that were significantly 
negatively related to $\mathrm{FiO}_{2}$ after $\mathrm{p}$ value adjustment. PC (18: 2_20: 4) was inversely correlated with PCT lever. Simultaneously, these five small molecule lipids were able to predict the length of hospital stay and 30-day mortality, they all can serve as the prognostic biomarker in patients with SCAP.

Lipids account for $90 \%$ of the surfactants in the lungs, and disorders of surfactants during pneumonia may cause changes in lipid metabolism [21]. Korneev, et al [22] reported that large amounts of lipid metabolites were produced by bacteria, and lipids were important for the structure and function of bacteria. At the same time, studies have shown that lipids are important inflammatory mediators during infection, and changes in lipid metabolites have been observed in cases such as sepsis [23], bacteraemia [24], and viral infections [25-26]. The differences in the lipid profile of HC, NSCAP, and SCAP group found in this study may be related to the above reasons. Therefore, it will be worthwhile to find meaningful lipid metabolites in biomarker discovery studies in the future. Arshad, et al [16] observed a striking decrease in the phospholipid concentrations in acute CAP, which largely normalized with clinical recovery. Thus, phospholipids can serve as highly accurate biomarkers for the diagnosis of CAP. This finding is consistent with our research. Simultaneously, decreased serum phospholipid concentrations have been documented in other invasive bacterial infections, such as bacteraemia and sepsis [27]. Studies by Arshad, et al [16] also confirmed that at least part of the observed reduction in plasma concentration might be pre-programmed at the level of infected cells due to the phospholipase activity. Obviously, additional studies are still needed to unravel the causes and consequences of reduced phospholipid abundance in CAP. Sphingolipids are an important part of the structure of biological membranes and has complex biological functions, such as mediating inflammation [28], regulating proliferation, signal transduction, and migration of cells, as well as apoptosis [29-30]. Previous studies have shown significant changes in sphingolipid metabolism pathways in BALF [9] and serum [31] in CAP patients. Imbalances in the sphingolipid metabolism pathways also occur in other lung inflammatory diseases, such as asthma [32] and chronic obstructive pulmonary disease [33]. In this study, we also observed significant changes in sphingolipid abundance in the serum of CAP patients. Therefore, targeting lipid metabolism pathways, including inhibiting ceramide synthesis and restoring sphingosine levels, might become a new treatment method for CAP, but needs further investigation. FA was detected only in the negative ion mode. Similarly, we found that compared with healthy controls, the abundance of FA in patients with CAP decreased and continued to decrease as the condition worsened. Studies have shown that the composition and concentration of FA are closely related to inflammation [34]. Saturated fatty acids can induce inflammation as pro-inflammatory lipids [35] and unsaturated fatty acids can inhibit the activation of inflammatory bodies [36]. Likewise, Sun et al [37] found that in the serum of patients with H7N9 pneumonia, the content of FA (16: 0$)$ gradually decreased with the increase in clinical symptoms of patients, and the levels increased after the patients' condition improved. Notably, biologically active lipids have complex regulatory mechanisms on inflammation in the body; this deserves further exploration.

Considering these intimate connections between lipids and processes related to the pathogenesis of pneumonia, it is conceivable that changes in plasma small lipid molecule can be mined for biomarker discovery. Biomarkers in body fluids can stimulate early identification of disease and severity 
assessment, which have been increasingly used in determining the diagnosis and predicting the prognosis in lung diseases $[6,15]$. Simultaneously, the discovery of new biomarkers in CAP and severe CAP also help predict treatment response and develop new insights into ongoing pathophysiological processes $[16,17]$. We therefore strictly limited the criteria to select the differential lipids in this study, based on the combination of VIP $>1$ and FDR $<0.05$, which was consistent with the methods used in some previous metabolomics studies [31, 38]. In combination with ROC analysis, 5 lipid small molecules were finally selected as potential biomarkers, which can distinguish between CAP group, healthy control group, and early identification of SCAP for subsequent research.

Lipids are involved in a variety of processes intimately connected with the pathogenesis of pneumonia and related infectious diseases [16]. PC is involved in the formation of immune synapses, and lipids play an important role in macrophage activation, NK cell function, differentiation, and activity of $\mathrm{T}$ and $\mathrm{B}$ effector cell [8,9]. Previous research noted that PC (16:0_18:1) was significantly up regulated following chlamydia trachomatis serovar $D$ infection in vivo and in vitro infection models. Exogenous addition of PC (16:0_18:1) resulted in a significant increase in chlamydia trachomatis serovar D in Hela 229 cells [39]. The finding of our study is similar to this of previous study [39].

Our analysis revealed several highly accurate plasma lipid biomarkers for CAP compared with $\mathrm{HC}$ and NSCAP with SCAP. The top 5 corresponded to PCs, which agreed well with the marked decrease observed in this class. We investigated the correlation between PCs abundance and a variety of clinical parameters. PC (18: 2_20: 4), $\mathrm{PC}$ (38: 6), and PC (36: 4) were negatively related to $\mathrm{FiO}_{2}$. In addition, $\mathrm{PaO}_{2}$ / $\mathrm{FiO}_{2} \geq 250$ can reflect the condition of hypoxemia in arteries, which is one of the important criteria for judging severe pneumonia. Plasma phospholipids merit further investigation as biomarkers not merely to reflect the pathophysiology in the patient's body, but that they also help monitor disease progression and early identify potential SCAP occurrences. Hence, PCT is currently considered an infection-related biomarker and can be used as a specific indicator of bacterial infection [40]. Remarkably, the degree of PCT elevation is closely related to bacterial load and infection severity [41], PC (18: 2_20: 4) was positively correlated with PCT lever in our study. The results did suggest that plasma phospholipids not only have strong CAP biomarker potential, they may also be closely related to bacterial infections. Through evaluating bacterial diseases, viruses, or other pathogen categories, it may help decide treatments to stop or gradually upgrade antibiotics. Langley et al [42] research showed profiles of specific metabolites measured on day 1 and 7 differed markedly between survivors and non-survivors of severe septic shock, and patients who did not survive within 90 days showed a marked decrease in PC species. Consistently, in this study we found that as the relative abundance of PC (18: 2_20: 4), PC (36: 4), PC (20: 5_18: 2), and PC (38: 6) decreased, the hospitalization days will be significantly extended. In particular, PC (16: 0_18: 1), PC (36: 4), and PC (20: 5_18: 2) decreased, and 30-day mortality rates increased significantly.

Our study is just an initial step in CAP severity assessment using a lipidomics approach. It has several limitations. Biomarker discovery is no small undertaking and requires validation testing to reach the application phase. Because we examined only 48 samples, our results might have been due to chance. 
The results must be confirmed in a large study. Similarly, the lack of effective independent external validation makes our initial findings ineffective. We should further design validation study to add tremendous validity to our initial findings. Furthermore, only serum lipid relative abundance was detected at the time of admission, dynamic and follow-up changes (in response to treatment) were not investigated. The effects of changes in lipid expression during the pathogenesis of CAP should be further investigated. In addition, we cannot clarify the specific role of potential biomarker lipids in the pathogenesis of CAP. This requires us to conduct larger sample cohort studies and more detailed mechanism studies. Multi-omics data were integrated into the CAP study to further support the development of precision medicine.

\section{Conclusions}

In conclusion, we demonstrated that lipidomics approaches based on HPLC-MS/MS could be successfully used to reveal lipids changes in CAP and establish a metabolite signature related to disease severity. The increase or decrease in the five molecular lipids in serum can serve as potential biomarkers of disease diagnosis, prognosis, and can accurately predict SCAP as well as significantly improve the 30day mortality prediction in patients with CAP.

\section{Abbreviations}




\begin{tabular}{|c|c|}
\hline Abbreviation & \\
\hline ANOVA & analysis of variance \\
\hline AUC & pneumonia severity index \\
\hline AUC & area under the curve \\
\hline BMI & body mass index \\
\hline CAP & community-acquired pneumonia \\
\hline CI & confidence interval \\
\hline COPD & chronic obstructive pulmonary disease \\
\hline CRP & C-reactive protein \\
\hline CURB-65 & CURB-65 confusion, urea, respiratory rate, blood pressure, and age $\geq 65$ years old \\
\hline CV-ANOVA & cross-validation analysis of variance \\
\hline $\mathrm{ESI}+/-$ & election spray ionization positive/negative mode \\
\hline FA & fatty acyls \\
\hline FDR & false discovery rate \\
\hline FFA & free fatty acid \\
\hline $\mathrm{FiO} 2$ & fraction of inspiration $\mathrm{O}_{2}$ \\
\hline GL & glycerlipid \\
\hline GP & glycerol-phospholipid \\
\hline $\mathrm{HC}$ & healthy control \\
\hline ICU & intensive care unit \\
\hline IQR & interquartile range \\
\hline LY & lymphocyte \\
\hline MLR & multiple linear regression \\
\hline MO & monocyte \\
\hline MVA & multivariate variable analysis \\
\hline $\mathrm{NE}$ & neutrophil \\
\hline NLR & neutrophil/lymphocyte ratio \\
\hline NSCAP & non-severe cap \\
\hline OPLS-DA & orthogonal partial least squares discriminant analysis \\
\hline $\mathrm{PaO}_{2}$ & partial pressure of oxygen, \\
\hline $\mathrm{PC}$ & phosphatidylcholine \\
\hline PCA & principal component analysis \\
\hline PCT & procalcitonin \\
\hline PKUPH & Peking University People's Hospital \\
\hline PLR & platelet-lymphocyte ratio \\
\hline PLT & blood platelet \\
\hline PR & prenol lipids \\
\hline PSI & pneumonia severity index \\
\hline QC & quality control \\
\hline ROC & receiver operating characteristic curve \\
\hline RT & retention time \\
\hline $\mathrm{SaO}_{2}$ & oxygen saturation \\
\hline SCAP & severe CAP \\
\hline SM & sphingomyelin \\
\hline SP & sphingolipid \\
\hline $\mathrm{ST}$ & sterol lipid \\
\hline UHPLC-MS/MS & ultra high-performance liquid chromatography with tandem mass spectrometry \\
\hline VIP & variable importance on projection \\
\hline WBC & white blood cell \\
\hline
\end{tabular}

\section{Declarations}

\section{Ethics approval and consent to participate}

The study protocol was approved by the Institutional Review Board of the Peking University People's Hospital (Beijing, China). Written informed consent was obtained from all patients or their surrogates.

\section{Consent for publication}


All the authors have read and approved the final manuscript and the manuscript is submitted solely to Critical Care.

\section{Availability of data and materials}

All data generated and/or analysed during this study are included in this published article and its supplementary information files.

\section{Competing interests}

The authors declare that they have no competing interests.

\section{Funding}

This study was funded by Chinese Science and Technology Key Project (2017ZX10103004-006)

\section{Authors' contributions}

Li Chen and Yali Zheng performed the experiments. Li Chen conducted the data collection, bioinformatics analyse, statistical analysis, interpretation and prepared the manuscript. Yali Zheng and Lili Zhao provided technical support during data analysis. Ying Zhang, Lu Yin, Yukun He and Xinqian Ma contributed substantially to the study design, samples and clinical data collection. Zhancheng Gao and Yu Xu had full access to all data in the study, contributed to the study concept and design, interpretation and drafting of the manuscript, andapproved the final manuscript. All authors read the manuscript and approved the submission.

\section{Acknowledgements}

The authors wish to thank staff members of the cooperating hospitals (Fujian Provincial Hospital, Sichuan University West China Hospital, Shanghai Pulmonary Hospital, The Second Hospital of Jinlin University and Tibet Autonomous Region People's Hospital) for assistance with samples and clinical data collection. They are also grateful to the Metabolomics Facility at the Technology Center for Protein Sciences, Tsinghua University for technical support.

\section{Contributor Information}

Li Chen, Email: bch_chenli2015@163.com

Yali Zheng, Email: yali_zheng@126.com

Lili Zhao, Email: zhaolily5352@163.com

Ying Zhang, Email: yingruoshui@163.com

Lu Yin, Email: yinludew@yeah.net 
Yukun He, Email: heyukun@bjmu.edu.cn

Xinqian Ma, Email: maxq@bjmu.edu.cn

Yu Xu, Email: pkuph_xuyu@163.com

Zhancheng Gao, Email: zcgao@bjmu.edu.cn

\section{References}

1. Wunderink RG, Waterer G. Advances in the causes and management of community acquired pneumonia in adults. BMJ.2017; 358:j2471.

2. Salluh JI, Soares M, Povoa P. Corticosteroids in severe community-acquired pneumonia: the path we choose depends on where we want to get. Crit Care. 2011; 15(2):137.

3. Chalmers JD. Identifying severe community-acquired pneumonia: moving beyond mortality. Thorax. 2015; 70(6):515-516.

4. Schuetz P, Wolbers M, Christ-Crain M, Thomann R, Falconnier C, Widmer I, Neidert S, Fricker T, Blum C, Schild $\mathrm{U}$ et al. Prohormones for prediction of adverse medical outcome in community-acquired pneumonia and lower respiratory tract infections. Crit Care. 2010; 14(3):R106.

5. Malloy JL, Wright JR. In vivo clearance of surfactant lipids during acute pulmonary inflammation. Respir Res. 2004; 5:8.

6. Christ-Crain M, Opal SM. Clinical review: the role of biomarkers in the diagnosis and management of community-acquired pneumonia. Crit Care. 2010; 14(1):203.

7. Cui L, Lee YH, Kumar Y, Xu F, Lu K, Ooi EE, Tannenbaum SR, Ong CN. Serum metabolome and lipidome changes in adult patients with primary dengue infection. PLoS Negl Trop Dis. 2013; 7(8):e2373.

8. Hannun YA, Obeid LM. Principles of bioactive lipid signalling: lessons from sphingolipids. Nat Rev Mol Cell Biol. 2008; 9(2):139-150.

9. Zheng Y, Ning P, Luo Q, He Y, Yu X, Liu X, Chen Y, Wang X, Kang Y, Gao Z. Inflammatory responses relate to distinct bronchoalveolar lavage lipidome in community-acquired pneumonia patients: a pilot study. Respir Res 2019; 20(1):82.

10. Guirgis FW, Donnelly JP, Dodani S, Howard G, Safford MM, Levitan EB, Wang HE. Cholesterol levels and long-term rates of community-acquired sepsis. Crit Care. 2016; 20(1):408.

11. Ryan AJ, McCoy DM, McGowan SE, Salome RG, Mallampalli RK. Alveolar sphingolipids generated in response to TNF-alpha modifies surfactant biophysical activity. J Appl Physiol (1985). 2003; 94(1):253-258.

12. Sharma NK, Tashima AK, Brunialti MKC, Ferreira ER, Torquato RJS, Mortara RA, Machado FR, Assuncao M, Rigato $\mathrm{O}$, Salomao R. Proteomic study revealed cellular assembly and lipid metabolism dysregulation in sepsis secondary to community-acquired pneumonia. Sci Rep. 2017; 7(1):15606. 
13. Filippas-Ntekouan S, Liberopoulos E, Elisaf M. Lipid testing in infectious diseases: possible role in diagnosis and prognosis. Infection. 2017; 45(5):575-588.

14. Iwabuchi K, Nakayama H, Oizumi A, Suga Y, Ogawa H, Takamori K. Role of Ceramide from Glycosphingolipids and Its Metabolites in Immunological and Inflammatory Responses in Humans. Mediators Inflamm. 2015; 2015:120748..

15. Mickiewicz B, Thompson GC, Blackwood J, Jenne CN, Winston BW, Vogel HJ, Joffe AR, Alberta Sepsis N. Development of metabolic and inflammatory mediator biomarker phenotyping for early diagnosis and triage of pediatric sepsis. Crit Care. 2015; 19:320.

16. Arshad H, Alfonso JCL, Franke R, Michaelis K, Araujo L, Habib A, Zboromyrska Y, Lucke E, Strungaru $\mathrm{E}, \mathrm{Akmatov} \mathrm{MK}$ et al. Decreased plasma phospholipid concentrations and increased acid sphingomyelinase activity are accurate biomarkers for community-acquired pneumonia. J Transl Med. 2019; 17(1):365.

17. To KK, Lee KC, Wong SS, et al. Lipid metabolites as potential diagnostic and prognostic biomarkers for acute community acquired pneumonia. Diagn Microbiol Infect Dis. 2016; 85(2):249-254.

18. Niederman MS, Mandell LA, Anzueto A, et al. Guidelines for the management of adults with community-acquired pneumonia. Diagnosis, assessment of severity, antimicrobial therapy, and prevention. Am J Respir Crit Care Med. 2001; 163(7):1730-1754.

19. Chong J, Soufan O, Li C, Caraus I, Li S, Bourque G, Wishart DS, Xia J. MetaboAnalyst 4.0: towards more transparent and integrative metabolomics analysis. Nucleic Acids Res. 2018; 46(W1):W486W494.

20. Boccard J, Rutledge DN. A consensus orthogonal partial least squares discriminant analysis (OPLSDA) strategy for multiblock Omics data fusion. Anal Chim Acta. 2013; 769:30-39.

21. Holčapek M, Liebisch G, Ekroos K. Lipidomic Analysis. Anal Chem. 2018; 90(7):4249-4257.

22. Korneev KV, Arbatsky NP, Molinaro A, Palmigiano A, Shaikhutdinova RZ, Shneider MM, Pier GB, Kondakova AN, Sviriaeva EN, Sturiale L et al. Structural Relationship of the Lipid A Acyl Groups to Activation of Murine Toll-Like Receptor 4 by Lipopolysaccharides from Pathogenic Strains of Burkholderia mallei, Acinetobacter baumannii, and Pseudomonas aeruginosa. Front Immunol. 2015; 6:595.

23. Golucci A, Marson FAL, Ribeiro AF, Nogueira RJN. Lipid profile associated with the systemic inflammatory response syndrome and sepsis in critically ill patients. Nutrition. 2018; 55-56:7-14.

24. To KK, Lee KC, Wong SS, et al. Lipid mediators of inflammation as novel plasma biomarkers to identify patients with bacteremia. J Infect. 2015; 70(5):433-444.

25. Manchester M, Anand A. Metabolomics: Strategies to Define the Role of Metabolism in Virus Infection and Pathogenesis. Adv Virus Res. 2017; 98:57-81.

26. Orchard RC, Wilen CB, Virgin HW. Sphingolipid biosynthesis induces a conformational change in the murine norovirus receptor and facilitates viral infection. Nat Microbiol. 2018; 3(10):1109-1114.

27. Drobnik W, Liebisch G, Audebert FX, Frohlich D, Gluck T, Vogel P, Rothe G, Schmitz G. Plasma ceramide and lysophosphatidylcholine inversely correlate with mortality in sepsis patients. J Lipid 
Res. $2003 ; 44(4): 754-761$.

28. Norris GH, Blesso CN. Dietary and Endogenous Sphingolipid Metabolism in Chronic Inflammation. Nutrients. 2017; 9(11).

29. Hannun YA, Obeid LM. Sphingolipids and their metabolism in physiology and disease [published correction appears in Nat Rev Mol Cell Biol. 2018 Oct;19(10):673]. Nat Rev Mol Cell Biol. 2018;19(3):175-191.

30. Albeituni S, Stiban J. Roles of Ceramides and Other Sphingolipids in Immune Cell Function and Inflammation. Adv Exp Med Biol. 2019; 1161:169-191.

31. Ning P, Zheng Y, Luo Q, Liu X, Kang Y, Zhang Y, Zhang R, Xu Y, Yang D, Xi W et al. Metabolic profiles in community-acquired pneumonia: developing assessment tools for disease severity. Crit Care. 2018; 22(1):130.

32. Perzanowski MS, Ono JG, Acosta LM, et al. Distinct Serum Sphingolipid Profiles among School-aged Children with Exercise-induced Wheeze and Asthma Persistence. Am J Respir Crit Care Med. 2017;195(8):1068-1070.

33. Bowler RP, Jacobson S, Cruickshank C, Hughes GJ, Siska C, Ory DS, Petrache I, Schaffer JE, Reisdorph N, Kechris K. Plasma sphingolipids associated with chronic obstructive pulmonary disease phenotypes. Am J Respir Crit Care Med. 2015; 191(3):275-284.

34. Fritsche KL. The science of fatty acids and inflammation. Adv Nutr. 2015; 6(3):293S-301S.

35. Glass CK, Olefsky JM. Inflammation and lipid signaling in the etiology of insulin resistance. Cell Metab. 2012; 15(5):635-645.

36. Yan Y, Jiang W, Spinetti T, et al. Omega-3 fatty acids prevent inflammation and metabolic disorder through inhibition of NLRP3 inflammasome activation. Immunity. 2013; 38(6):1154-1163.

37. Sun X, Song L, Feng S, et al. Fatty Acid Metabolism is Associated With Disease Severity After H7N9 Infection. EBioMedicine. 2018;33:218-229.

38. Cao B, Wang D, Pan Z, Brietzke E, Mclntyre RS, Musial N, Mansur RB, Subramanieapillai M, Zeng J, Huang $\mathrm{N}$ et al. Characterizing acyl-carnitine biosignatures for schizophrenia: a longitudinal pre- and post-treatment study. Transl Psychiatry. 2019; 9(1):19.

39. Wali S, Gupta R, Yu JJ, et al. Guinea pig genital tract lipidome reveals in vivo and in vitro regulation of phosphatidylcholine 16:0/18:1 and contribution to Chlamydia trachomatis serovar D infectivity. Metabolomics. 2016; 12(4):74.

40. Simon L, Gauvin F, Amre DK, Saint-Louis P, Lacroix J. Serum procalcitonin and C-reactive protein levels as markers of bacterial infection: a systematic review and meta-analysis. Clin Infect Dis. 2004; 39(2):206-217.

41. Sager R, Kutz A, Mueller B, Schuetz P. Procalcitonin-guided diagnosis and antibiotic stewardship revisited. BMC Med. 2017; 15(1):15.

42. Langley RJ, Tsalik EL, van Velkinburgh JC, Glickman SW, Rice BJ, Wang C, Chen B, Carin L, Suarez A, Mohney RP et al. An integrated clinico-metabolomic model improves prediction of death in sepsis. 
Sci Transl Med. 2013; 5(195):195ra195.

\section{Tables}


Table 1. Demographic, clinical and laboratory characteristics of the subjects enrolled in this study

\begin{tabular}{|c|c|c|c|c|}
\hline Characteristic & $\begin{array}{c}\text { SCAP } \\
(\mathrm{N}=13)\end{array}$ & $\begin{array}{l}\text { NSCAP } \\
(\mathrm{N}=15)\end{array}$ & $\begin{array}{c}\mathrm{HC} \\
(\mathrm{N}=20)\end{array}$ & $p$ value \\
\hline Male sex - no. (\%) & $8(61.5)$ & $6(40.00)$ & $7(35.0)$ & 0.304 \\
\hline Age - years & $63.9 \pm 19.284$ & $67.36 \pm 16.782$ & $41.67 \pm 15.144$ & 0.279 \\
\hline $\mathrm{BMI}-\left(\mathrm{kg} / \mathrm{m}^{2}\right)$ & $23.65 \pm 3.200$ & $20.79(17.31-22.87)$ & $19.91 \pm 2.504$ & 0.133 \\
\hline Smoking history — no. (\%) & $4(30.80)$ & $5(33.30)$ & $3(15.00)$ & 0.396 \\
\hline Smoking index & $0(0-14.50)$ & $0(0-10.00)$ & $0(0-0)$ & 0.533 \\
\hline \multicolumn{5}{|l|}{ Underlying diseases } \\
\hline COPD & $0 \square 0 \square$ & $106.70 \square$ & $0 \square 0 \square$ & 0.325 \\
\hline Asthma & $2 \square 15.40 \square$ & $1 \square 6.70 \square$ & $0 \square 0 \square$ & 0.203 \\
\hline Bronchiectasis & $0 \square 0 \square$ & $1 \square 6.70 \square$ & $0 \square 0 \square$ & 0.325 \\
\hline Interstitial lung Disease & $1 \square 7.70 \square$ & $0 \square 0 \square$ & $0 \square 0 \square$ & 0.253 \\
\hline Hypertension & $4 \llbracket 30.80 \square$ & $5 \square 33.30 \square$ & $5 \square 25 \square$ & 0.856 \\
\hline Cardiovascular disease & $3 \square 23.10 \square$ & $0 \square 0 \square$ & $4 \square 20 \square$ & 0.151 \\
\hline Cerebrovascular disease & $1 \square 7.70 \square$ & $0 \square 0 \square$ & $2 \square 10 \square$ & 0.466 \\
\hline Cardiac insufficiency & $1 \square 7.70 \square$ & $3 \llbracket 13.30 \square$ & $0 \square 0 \square$ & 0.264 \\
\hline Autoimmune disease & $1 \square 7.70 \square$ & $0 \square 0 \square$ & $0 \square 0 \square$ & 0.253 \\
\hline \multicolumn{5}{|l|}{ Physical examination } \\
\hline $\mathrm{T} \operatorname{Max}\left({ }^{\circ} \mathrm{C}\right)$ & $38.63 \pm 1.48$ & $37.78 \pm 1.22$ & NA & 0.125 \\
\hline Respiratory frequency (times / min) & $22(20-24)$ & $20(20-21)$ & NA & 0.025 \\
\hline Systolic pressure $(\mathrm{mmHg})$ & 129.50(113-149) & $120(115-128)$ & NA & 0.957 \\
\hline Diastolic blood pressure (mmHg) & $74 \pm 14.09$ & $72.60 \pm 10.03$ & NA & 0.765 \\
\hline Mean arterial pressure (mmHg) & $91.06 \pm 16.48$ & $89.93 \pm 8.18$ & NA & 0.832 \\
\hline Lung rales - no. (\%) & 10(76.92) & $9(60.00)$ & NA & 0.435 \\
\hline Disorder of consciousness & $3(23.08)$ & $0 \square 0 \% \square$ & NA & 0.087 \\
\hline \multicolumn{5}{|l|}{ Laboratory results } \\
\hline $\mathrm{WBC}\left(\times 10^{9} / \mathrm{L}\right)$ & $13.68 \pm 8.64$ & $6.38 \pm 2.48$ & NA & 0.030 \\
\hline $\mathrm{NE}\left(\times 10^{9} / \mathrm{L}\right)$ & $12.05 \pm 8.40$ & $3.96 \square 2.90-4.57 \square$ & NA & 0.008 \\
\hline $\mathrm{LY}\left(\times 10^{9} / \mathrm{L}\right)$ & $0.95 \pm 0.50$ & $1.46 \pm 0.56$ & NA & 0.022 \\
\hline $\mathrm{MO}\left(\times 10^{9} / \mathrm{L}\right)$ & $0.47(0.23-0.78 \square$ & $0.42 \square 0.27-0.65 \square$ & NA & 0.571 \\
\hline NE percentages $(\%)$ & $84.72 \pm 8.12$ & 60(57.20-65.60) & NA & $<0.0001$ \\
\hline LY percentages (\%) & $10.05 \pm 6.38$ & $29.60(19.90-31.60)$ & NA & $<0.001$ \\
\hline MO percentages (\%) & $4.05(2.45-5.60)$ & $7.88 \pm 3.07$ & NA & $<0.01$ \\
\hline NLR $(\%)$ & $10.75(4.48-20.58)$ & $2.20(1.80-4.90)$ & NA & 0.054 \\
\hline PLR (\%) & $265.45(122.58-410.60)$ & 144.70(123.50-199.10) & NA & 0.151 \\
\hline $\operatorname{PLT}\left(\times 10^{9} / \mathrm{L}\right)$ & $228.39 \pm 110.77$ & $216 \pm 68.90$ & NA & 0.774 \\
\hline $\mathrm{CRP}(\mathrm{mg} / \mathrm{L})$ & 63(9.075-76.625) & $95.995(27.125-259)$ & NA & 0.018 \\
\hline PCT $(\mu \mathrm{g} / \mathrm{L})$ & $0.115(0.038-0.37)$ & $0.65(0.285-6.168)$ & NA & 0.033 \\
\hline $\mathrm{PaO}_{2}(\mathrm{mmHg})$ & $73.208 \pm 19.268$ & $80(49.5-95.4)$ & NA & 0.978 \\
\hline $\mathrm{FiO}_{2}(\%)$ & 33(29-52.5) & 21(21-21) & NA & 0.004 \\
\hline $\mathrm{PaO}_{2} / \mathrm{FiO}_{2}$ & $199.89 \pm 66.86$ & $377.71 \pm 47.94$ & NA & $<0.0001$ \\
\hline $\mathrm{PaCO}_{2}(\mathrm{mmHg})$ & $39.83 \pm 10.32$ & $36.06 \pm 4.51$ & NA & 0.273 \\
\hline $\mathrm{SaO}_{2}(\%)$ & $95.30 \pm 2.47$ & $97.07 \pm 1.10$ & NA & 0.875 \\
\hline $\mathrm{HCO}_{3}(\mathrm{mmol} / \mathrm{L})$ & $24.98 \pm 6.06$ & $24 \pm 2.40$ & NA & 0.580 \\
\hline \multicolumn{5}{|l|}{ Detected pathogen } \\
\hline Bacteria-no. (\%) & $7(53.85)$ & $2(13.33)$ & NA & 0.042 \\
\hline Virus-no. (\%) & $2(15.38)$ & 8(53.33) & NA & 0.055 \\
\hline Fungus- no. (\%) & $4(30.77)$ & $5(33.33)$ & NA & 1.000 \\
\hline CURB-65 & $2(1-2)$ & $1(0-1)$ & NA & 0.010 \\
\hline PSI & $91.17 \pm 28.22$ & $67.40 \pm 30.51$ & NA & 0.027 \\
\hline
\end{tabular}


Abbreviations: BMI body mass index, COPD chronic obstructive pulmonary disease, WBC white blood cell, NE neutrophil, LY lymphocyte, MO monocyte, NLR neutrophil/lymphocyte ratio, PLR platelet-lymphocyte ratio, PLT blood platelet, CRP C-reactive protein, PCT procalcitonin, $\mathrm{PaO}_{2}$ partial pressure of oxygen, $\mathrm{FiO}_{2}$ Fraction of inspiration $\mathrm{O}_{2}, \mathrm{SaO}_{2}$ oxygen saturation, CURB-65 confusion, urea, respiratory rate, blood pressure, and age $\geq 65$ years old, PSI pneumonia severity index, NA not applicable.

Table 2. Evaluation parameters of our OPLS-DA model

\begin{tabular}{cccccccc}
\hline \multirow{2}{*}{ Group } & \multicolumn{3}{c}{ Evaluation parameters of OPLS-DA original model } & \multicolumn{2}{c}{ Permutation test } \\
\cline { 2 - 8 } & PC $^{\text {a }}$ & R2X & R2Y & Q2(cum) & CV-ANOVA $(p)$ & R2 & Q2 \\
\hline CAP vs HC & $1+4+0$ & 0.476 & 0.979 & 0.685 & 0.000001 & 0.913 & -0.566 \\
NSCAP vs HC & $1+2+0$ & 0.329 & 0.947 & 0.703 & 0.00000257 & 0.845 & -0.489 \\
SCAP vs HC & $1+2+0$ & 0.448 & 0.964 & 0.848 & 0.00000000172 & 0.790 & -0.509 \\
NSCAP vs SCAP & $1+2+0$ & 0.366 & 0.964 & 0.536 & 0.00757053 & 0.847 & -0.386 \\
\hline
\end{tabular}

a: PC The number of principal components in this model, which represents the number of predicted components plus the number of orthogonal components

CAP community-acquired pneumonia, HC healthy control, NSCAP non-severe CAP, SCAP severe CAP

\section{Additional Files}

Additional file 1: Supplemental Figures. Figure S1. The permutation test of the OPLS-DA model constructed by CAP versus $\mathrm{HC}$ a, NSCAP versus $\mathrm{HC} b$, SCAP versus $\mathrm{HC} c \mathrm{c}$ and SCAP versus SCAP $d$. All OPLS-DA models have been verified by permutation test. Permutation verification established after 500 iterations. The permutations plot helps to assess the risk that the current OPLS-DA model is spurious. The plot shows, for a selected Y-variable, on the vertical axis the values of R2 and Q2 for the original model (far to the right) and of the Y-permuted models further to the left. The horizontal axis shows the correlation between the permuted $\mathrm{Y}$-vectors and the original Y-vector for the selected $\mathrm{Y}$. The plot above strongly indicates that the original model is valid. The criteria for validity are: all blue Q2-values to the left are lower than the original points to the right or the blue regression line of the Q2-points intersects the vertical axis (on the left) at, or below zero. Figure S2. ROC analysis of potential biomarkers for differentiating NSCAP patients from SCAP. Comparision of ROC curves between five potential biomarkers and CURB-65 a, PSI b. PC(16:0_18:1), PC(18:2_20:4), PC(20:5_18ه2)『PC(36:4) and PC(38:6) showed the AUC of 0.933 (95\% Cl: 0.772-0.933), 0.954 (95\% Cl: 0.801-0.998), 0.872 (95\% Cl: 0.691-0.967), 0.877 (95\% Cl: 0.698-0.970), and 0.959 (95\% Cl: 0.808-0.998), respectively. The CURB-65 presented an AUC of 0.772 (95\% Cl: $0.575-0.908)$ and the PSI achieved an AUC of 0.749 (95\% Cl: $0.550-0.892)$. Figure S3. Correlation between putative biomarkers and clinical indicators. The color transition from dark blue to red indicates the correlation from low to high.

Additional file 2: Supplemental Tables. Table S1. Correlation analysis between five lipids for CAP and clinical indicators -Pearson's correlation coefficient. Table S2. Correlation analysis between five lipids for CAP and clinical indicators- $P$ value 
a

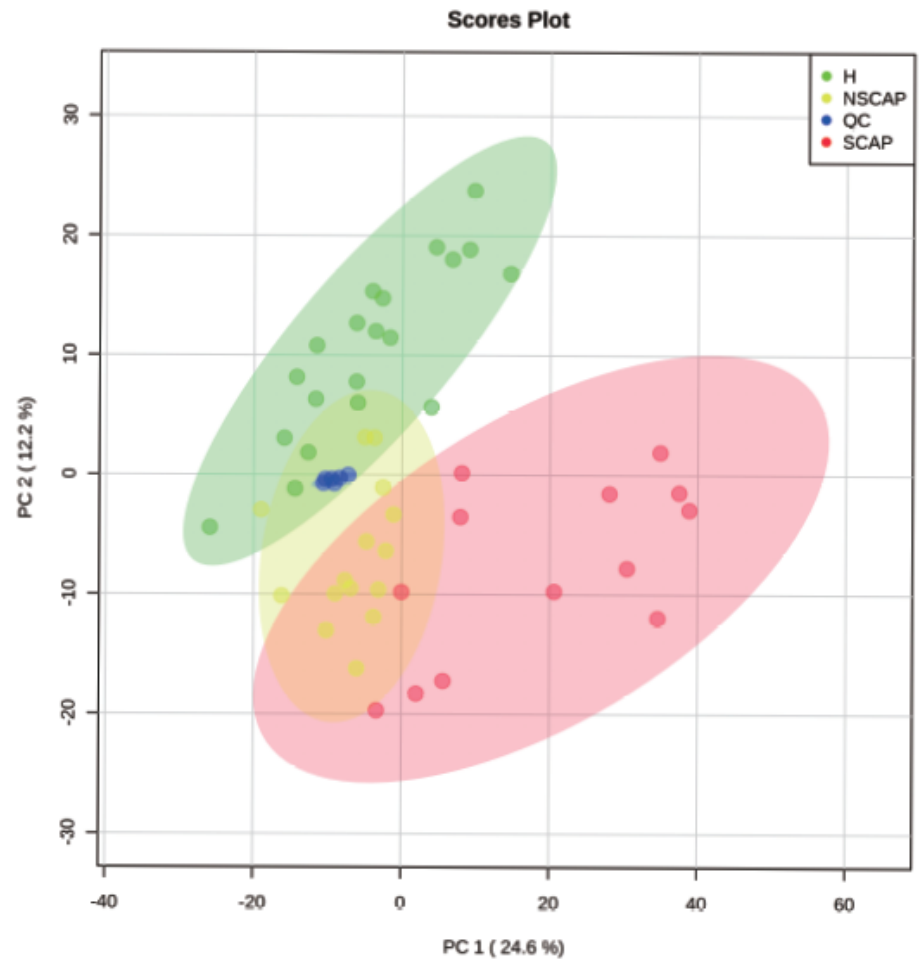

b

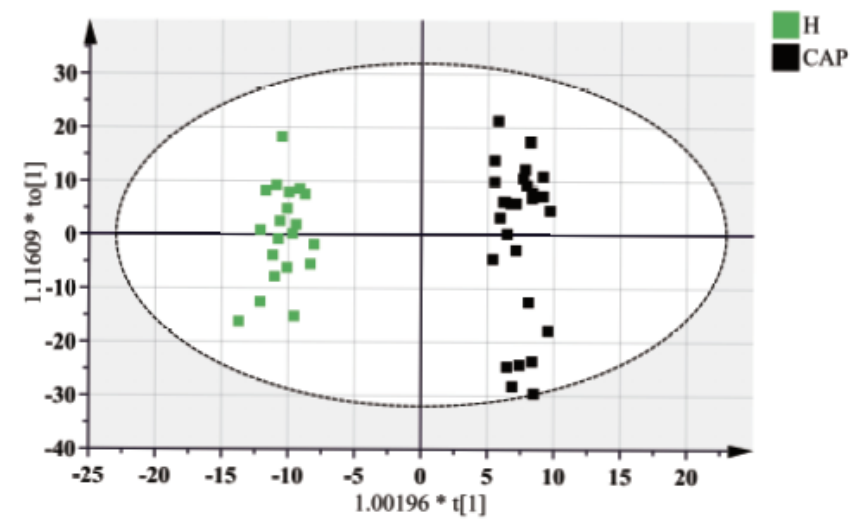

c

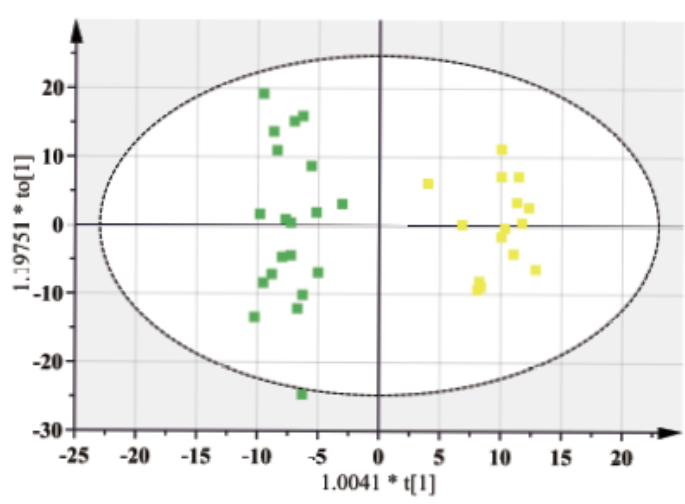

H

NSCAP

d

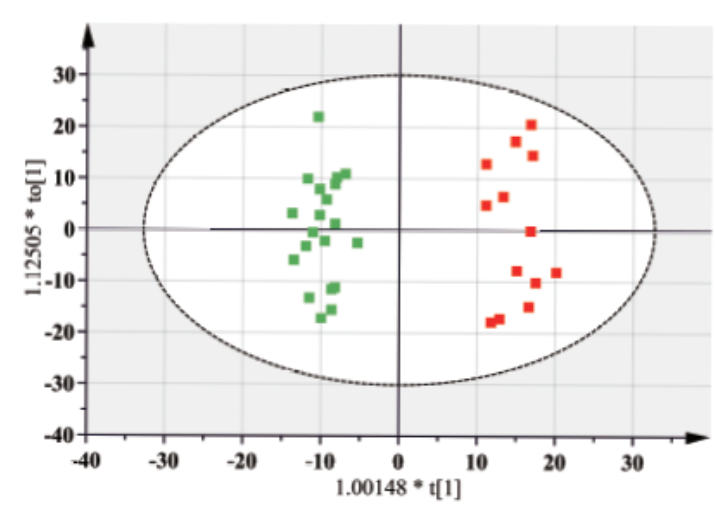

e

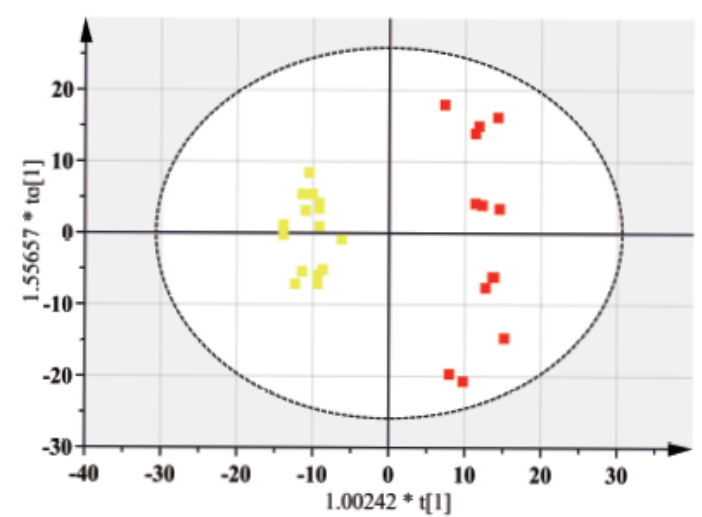

Figure 1

Multivariate statistical analysis of serum samples in CAP group (NSCAP and SCAP) and HC. a PCA scores plot of lipidomic profiles. Red, severe CAP (SCAP); yellow, non-severe CAP (NSCAP); green, healthy control (HC); and blue, quality control (QC) samples. No sample was placed outside the ellipse that describes the $95 \% \mathrm{Cl}$ of Hotelling's T-squared distribution. The three groups of lipid profiles can be clearly distinguished. b OPLS-DA score plot discriminates all CAP subjects versus HC. c OPLS-DA score plots of 
NSCAP versus HC group. d OPLS-DA score plots of SCAP versus HC. e OPLS-DA score plots of NSCAP versus SCAP. The PCA and OPLA-DA model reflect good separation trends among SCAP, NSCAP and HC.

a

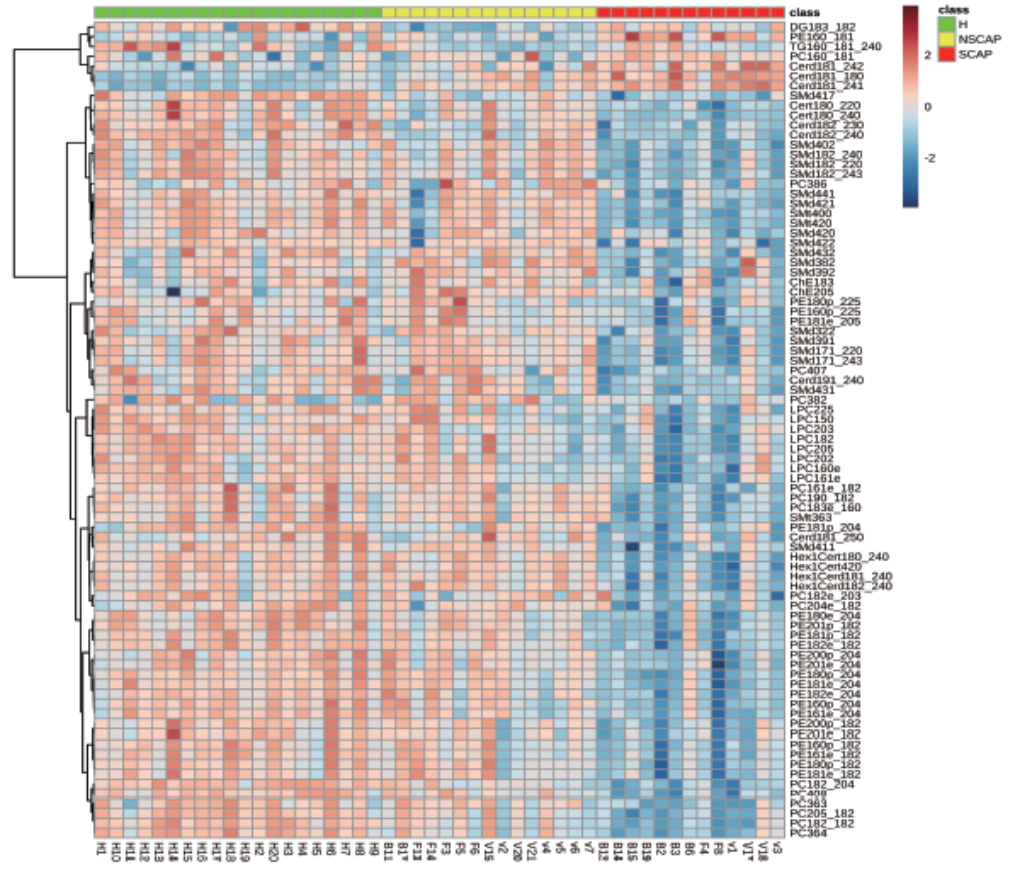

d

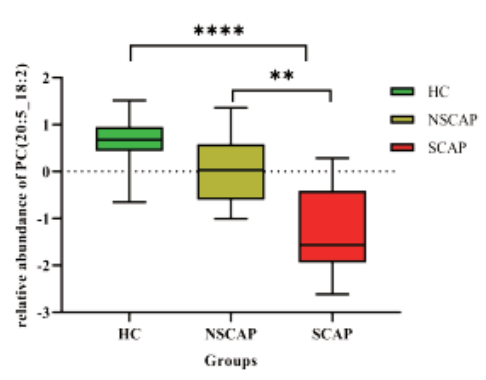

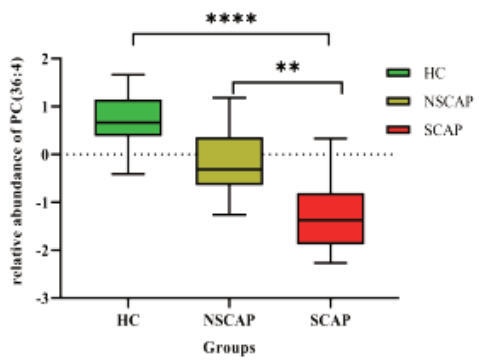

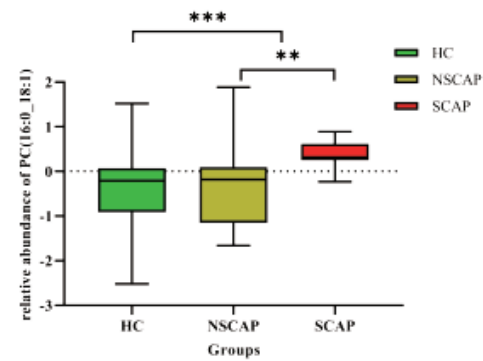

c

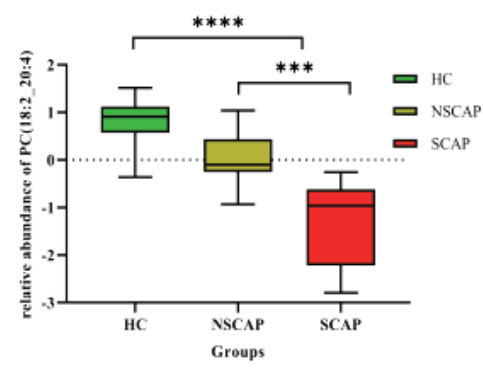

f

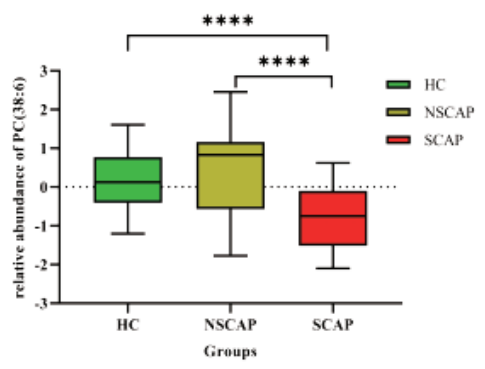

\section{Figure 4}

A great number of lipids dysregulated in three groups. a Hierarchical cluster heatmap of 85 lipids in CAP 刃including NSCAP and SCAP『compared to HC. Distance mearsure: Pearson; Clustering algorithm: ward. Row represents lipids and column represents serum samples. Green, yellow and red represent HC, NSCAP, and SCAP, respectively. Light blue indicates lower relative abundance, while greater brown indicates higher intensity of lipids. b-f The relative abundance of PC(16:0_18:1), PC(18:2_20:4), PC(20:5_18区2) $\mathrm{PC}(36 \varangle 4)$ and $\mathrm{PC}(38: 6)$ in serum between CAP group versus HC and NSCAP versus SCAP group were upregulated or down-regulated. ${ }^{*} p<0.05,{ }^{*} p<0.001,{ }^{\star \star *} p<0.001$, ${ }^{\star \star \star \star} p<0.0001$. 
a

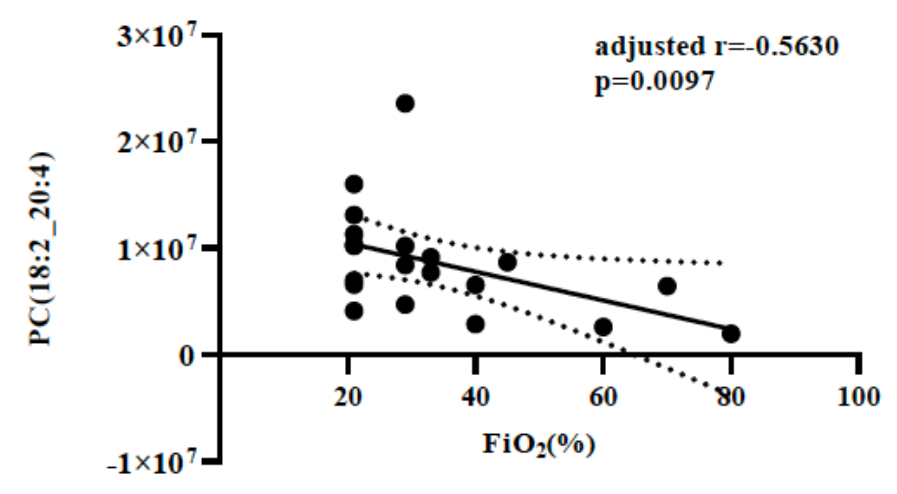

c

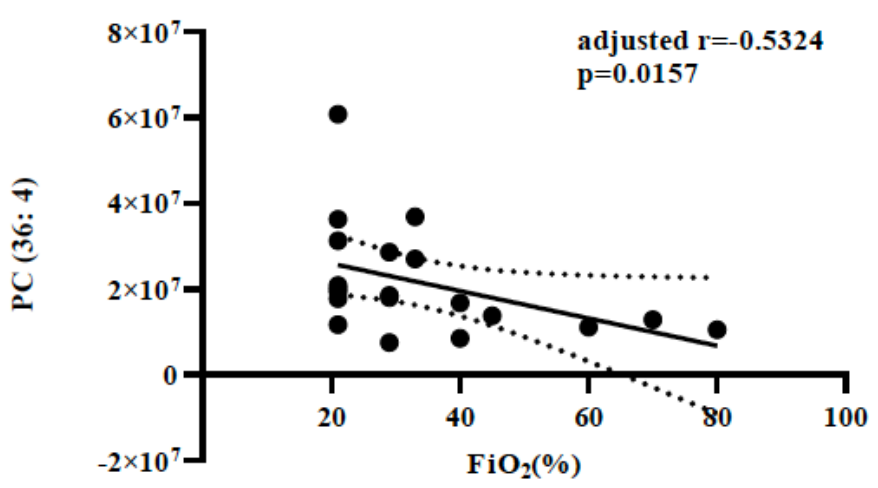

b

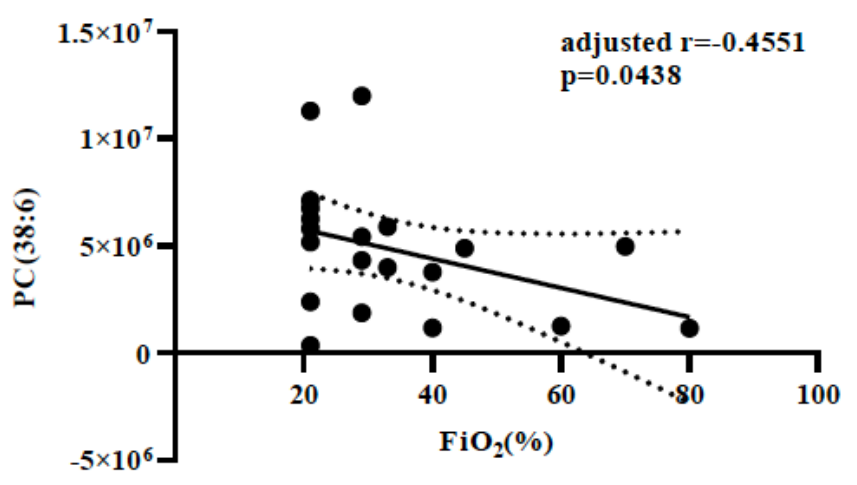

d

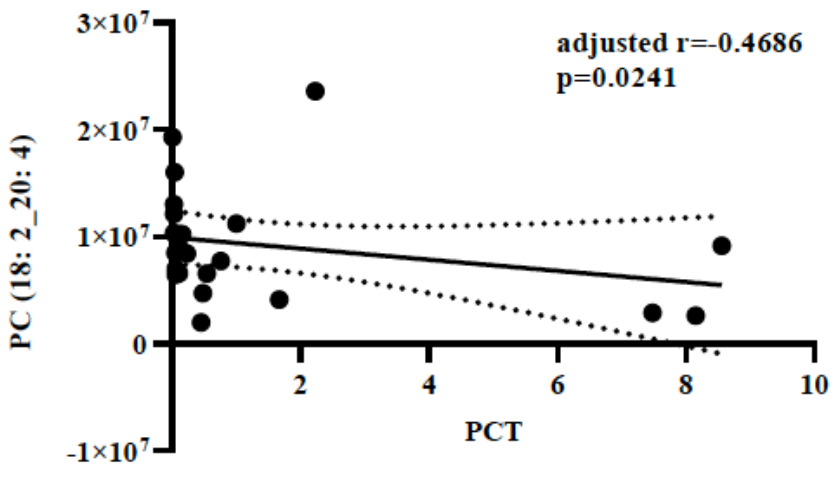

Figure 6

Three target lipid species show significantly correlations with clinical indicators. a-c PC(18:2_20:4), PC(38:6), and PC(36:4) are inversely correlated to FiO2 of CAP patients. d PC (18:2_20:4) is negatively correlated with PCT of CAP patients. Solid black line, the fitted regression line. Areas within the dotted line lines, the $95 \%$ confidence intervals. 


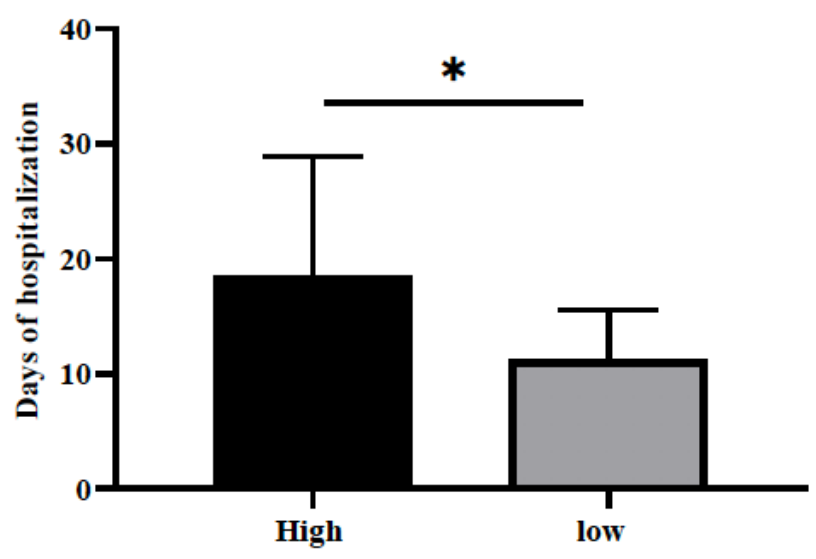

PC(16:0_18:1) relative abundance group

c

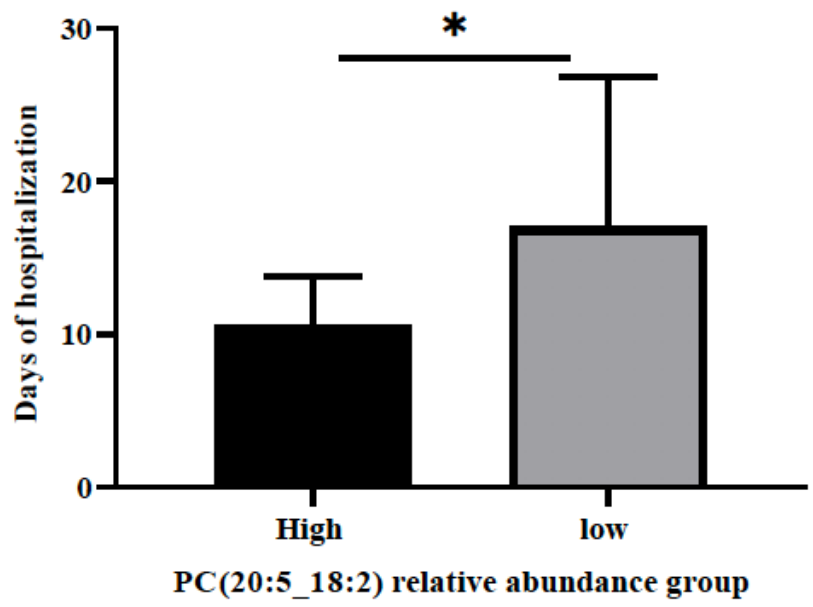

b

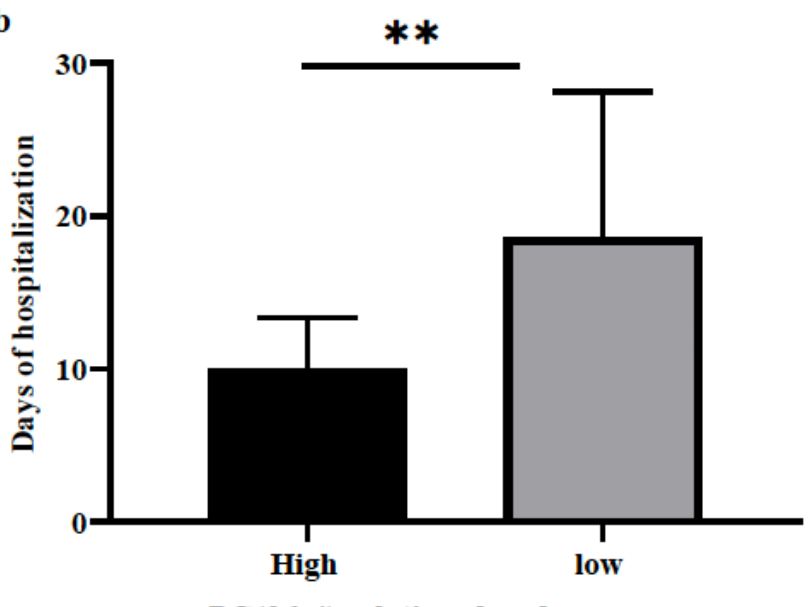

$\mathrm{PC}(36: 4)$ relative abundance group

d

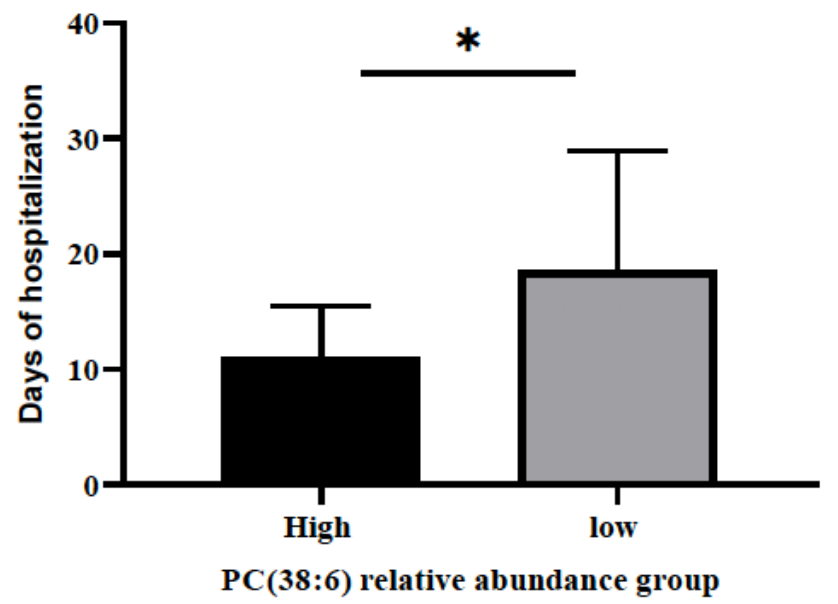

Figure 8

Compared the hospitalization days between the higher abundance and lower abundance group of 4 target lipids. a-d There were statistical differences in hospitalization days between two groups of $P C\left(16: 0 \_18: 1\right), P C(36 \varangle 4), P C\left(20: 5 \_18 \varangle 2\right)$, and $P C(38: 6)$. Black high abundance group, gray low abundance group. * $p<0.05, * \star p<0.001$ 

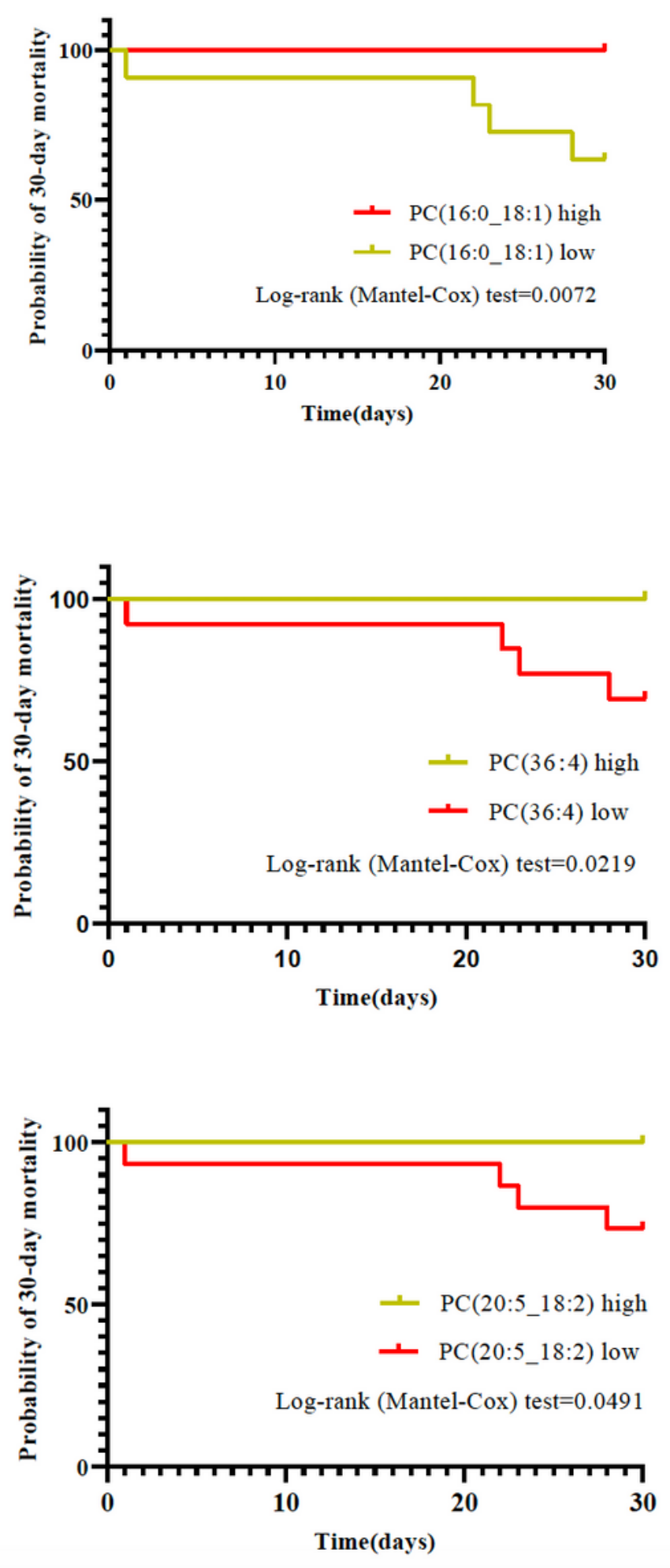

\section{Figure 10}

Kaplan-Meier analysis of 30-day mortality in patients with CAP. Analysis was stratified by target lipids relative abundance level. There were statistically significant difference in 30-day mortality between the high-abundance group and the low-abundance group of PC (16: 0_18: 1) a, PC (36: 4) b and PC (20: 5_18: 2) c. 


\section{Supplementary Files}

This is a list of supplementary files associated with this preprint. Click to download.

- FigureS2.pdf

- FigureS3.tiff

- TableS1.xIsx

- Tables2.xlsx

- Figures2.pdf

- FigureS3.tiff

- TableS1.xlsx

- TableS2.xIsx

- FigureS1.pdf

- FigureS1.pdf 